\title{
AN ATTEMPT TO ESTIMATE FIRING TEMPERATURE USING OSL PRE-DOSE SENSITIZATION OF QUARTZ
}

\author{
DILEEP K. KOUL ${ }^{1}$ and MOHAN P. CHOUGAONKAR ${ }^{2}$ \\ ${ }^{l}$ Astrophysical Sciences Division, Bhabha Atomic Research Centre, Mumbai- 400 085, India \\ ${ }^{2}$ Environmental Assessment Division, Bhabha Atomic Research Centre, Mumbai- 400 085, India
}

Received 5 February 2010

Accepted 8 September 2010

\begin{abstract}
The estimation of firing temperature of ceramics using methods based on pre-dose sensitization of the $110^{\circ} \mathrm{C}$ TL peak of quartz have yielded incoherent results. Recently the fast component of the CW-OSL of quartz has been observed to have pre-dose characteristics similar to that of the $110^{\circ} \mathrm{C}$ TL peak. The motivation for this study has been to look into the feasibility of this signal, the OSL counter part of pre-dosed TL emission, for the firing temperature identification. The variation in the sensitization ratio of the TL and OSL emissions with different heating temperatures was also utilized to unveil the firing temperature mark of the specimen.
\end{abstract}

Keywords: TL, OSL, firing, quartz, sensitization.

\section{INTRODUCTION}

Thermal treatment has a profound influence on luminescence properties of a phosphor. The luminescence dating methods incorporate the pre-heating or heat depletion in their protocols (Aitken, 1985). The sensitivity change experienced by the sample since antiquity is primarily governed by the thermal treatment experienced by the sample during zeroing episode or thereafter during its lifetime since zeroing event (Roque et al., 2004). As perfect zeroing is a pre-requisite for reliability of $\mathrm{TL}$ dating technique, the information about the firing temperature, prevailing during zeroing episode, will lend reliability to the age estimation of the ceramic.

Various non-luminescent techniques have been applied to see the impact of heat treatment on archaeological and geological specimens. The effect of heating; (i) on the broadening of diffraction lines (Weymouth and Mandeville, 1975), (ii) electron resonance signal (Robins et al., 1978) and (iii) Mossbauer spectrum (Jones et al., 1979) have been studied to monitor the firing temperature.

Corresponding author: D.K. Koul

e-mail: dkkoul@barc.gov.in
Methods based on luminescence phenomenon have been proposed for various natural phosphors. Valladas (1983) proposed a method for flint based on the properties of the $380^{\circ} \mathrm{C}$ emission. Göksu et al. (1989) and Godfrey-Smith and Ilani (2004) utilized the sensitization of the $110^{\circ} \mathrm{C}$ peaks in flint to record the temperatures experienced by these samples in the past. Lahaye et al. (2006) devised a method based on the thermal activation characteristics of the $110^{\circ} \mathrm{C}$ peak of quartz to unveil the thermal history of ferruginous sandstones. Polymeris et al. (2007) suggested that techniques based on both luminescence emissions, TL and OSL, would be able to provide an assessment of the firing temperature.

The pre-dose sensitization of the crystalline quartz is governed by the previous radiation dose and the subsequent thermal activation experienced by it. Zimmerman established the pre-dose model based on the transfer of charges from one recombination centre to another (Zimmerman, 1971). Attempts have been made to estimate the firing temperature of quartz using methods based on the TL pre-dose sensitization luminescence (Sunta and David, 1982; Watson and Aitken, 1985; Koul et al, 1996). The proposed method involves studying the behaviour of 
the pre-dose sensitivity of the pre-fired quartz with the subsequent annealing at different increasing temperatures, called re-firing temperatures. The findings of these groups reached different conclusions. While Sunta and David (1982) firmly established the authenticity of the method Watson and Aitken (1985) and Koul et al., (1996) found that the procedure might not be generally applicable.

The nature of the $110^{\circ} \mathrm{C}$ TL peak of quartz and the fast component of the CW-OSL has been observed to be very similar in nature (Aitken and Smith, 1988; Murray and Roberts, 1998). Based on the correlation studies, it is now widely believed that the two processes share the same luminescence centres (Franklin et al., 1995; Chen et al., 2001). Recently the characterization of the pre-dose phenomenon, a special feature of the $110^{\circ} \mathrm{C}$ TL peak, of the fast component of the CW-OSL suggested that it also possesses the signatures of the pre-dose phenomenon (Koul and Chougaonkar, 2007). The pre-dose behaviour of the two luminescence emissions has been observed to be very similar.

The relationship between $110^{\circ} \mathrm{C}$ TL and OSL emissions of quartz has been reported to be influenced by the firing temperature (Murray and Wintle, 2000; Chen et al. 2000). In the development of the single-aliquot regenerative-dose (SAR) protocol for OSL dating the $110^{\circ} \mathrm{C} \mathrm{TL}$ peak was found to be proportional to the OSL signal till a thermal treatment of $500^{\circ} \mathrm{C}$. Moreover, heating beyond this temperature was also observed to favour the TL process. To explain this behaviour Li (2002) proposed a model which proposed that the TL and OSL emission processes are related to different suites of the R-centres. This disparity between the two signals has been suggested to might occur also due to (i) difference in the capture probability of the $110^{\circ} \mathrm{C} \mathrm{TL}$ and OSL traps in charge capture during irradiation process and (ii) the variation in the degree of competition offered by the $\mathrm{E}_{1}$ ' centre, one of the main defect centres present in quartz, to the electrons released from these two traps during the luminescence read out (Koul, Submitted).

This study was motivated by the above mentioned two features (i) observation of the pre-dosed OSL signal and (ii) influence of high temperature heating on the TL/OSL ratio. Accordingly, procedures based on the observation of these two phenomena of the pre-fired samples with increasing heating temperatures, re-firing, were undertaken to identify the firing temperature of quartz specimen.

\section{EXPERIMENTAL DETAILS}

Quartz grains, 90-125 $\mu \mathrm{m}$, were extracted from two local geological samples, (i) Thar desert, India and (ii) municipal sludge, Baroda, India using conventional method. The sludge is being irradiated in a radio-isotope plant at SERI, Baroda, India. The heat treatments were performed in a resistance furnace for one hour in air for all the samples. Thereafter, the samples were allowed to cool down in the furnace itself. All the data points in the study contain the average value of three observations carried out using three fresh discs. Disc to disc reproducibility of better than 5\% was observed in case of most of the measurements. The heating rate during TL measurements was set at $5^{\circ} \mathrm{C} / \mathrm{s}$. The OSL signal was represented by the initial signal recorded during the first $0.16 \mathrm{~s}$.

Luminescence measurements were made using an automatic Risø TL/OSL system, TL-DA-15. It can accommodate 48 samples and has an on-plate attached $\beta$-irradiator. It provides a cluster of 49 blue light emitting diodes delivering a power of $\sim 25 \mathrm{~mW} / \mathrm{cm}^{2}$ to the sample. The detection filter used in all observations was a Hoya U-340 $\left(\mathrm{I}_{\mathrm{p}} \sim 340 \mathrm{~nm}, \mathrm{FWHM} \sim 80 \mathrm{~nm}\right)$. Irradiation of the samples was carried out using a ${ }^{90} \mathrm{Sr} /{ }^{90} \mathrm{Y} \beta$-source (dose rate $1.39 \mathrm{~Gy} / \mathrm{min}$ ) housed in the system.

\section{OBSERVATIONS}

The measurements in this study essentially involved looking into the nature of luminescence of the pre-fired samples with re-firing at different temperatures. The various features observed in this work are as under.

\section{Behaviour of pre-dose sensitization of pre-fired sam- ples with re-firing}

The samples, Thar and Sludge, were fired at temperatures of 600 and $800^{\circ} \mathrm{C}$ respectively for one hour in air. These samples will be referred to as pre-fired samples. The sub-samples derived from them were subsequently re-fired at different temperatures 400 to $900^{\circ} \mathrm{C}$ and 400 to $1000^{\circ} \mathrm{C}$ in steps of $100^{\circ} \mathrm{C}$ for Thar and Sludge samples respectively. The slightly higher upper window of refiring temperature for the latter was chosen due to the fact that it has been fired at higher temperature, $800^{\circ} \mathrm{C}$. The idea was to have sufficient number of data points around the firing temperature mark. The sensitization measurements of the $110^{\circ} \mathrm{C} \mathrm{TL}$ and OSL emissions were measured using the procedure as follows:

1) Administer a test dose (0.4 Gy).

2) Pre-heat at $160^{\circ} \mathrm{C}$ and record $110^{\circ} \mathrm{C} \mathrm{TL}$ peak $\left(\mathrm{TL}_{\mathrm{o}}\right)$.

3) Stimulate for $40 \mathrm{~s}$ at $125^{\circ} \mathrm{C}$ to record OSL $\left(\mathrm{OSL}_{\mathrm{o}}\right)$.

4) Administer a laboratory dose - pre-dose (10 Gy).

5) Heat the sample in glow oven at $380^{\circ} \mathrm{C}$ (activation temperature) in a usual way like recording a glow curve.

6) Repeat steps (1) to (3) to record $110^{\circ} \mathrm{C} \mathrm{TL}\left(\mathrm{TL}_{\beta}\right)$ and OSL $\left(\mathrm{OSL}_{\beta}\right)$ signals.

$\mathrm{TL}_{\mathrm{o}}$ and $\mathrm{OSL}_{\mathrm{o}}$ represent the test dose signals of $110^{\circ} \mathrm{C}$ $\mathrm{TL}$ and fast component of CW-OSL, recorded prior to the application of the pre-dose treatment respectively, while as, $\mathrm{TL}_{\mathrm{o}}$ and $\mathrm{OSL}_{\mathrm{o}}$ represent the signals after the application of the pre-dose treatment. The sensitization was defined as $\left(\mathrm{TL}_{\beta} / \mathrm{TL}_{\mathrm{o}}\right)$ and $\left(\mathrm{OSL}_{\beta} / \mathrm{OSL}_{\mathrm{o}}\right)$ for $\mathrm{TL}$ and $\mathrm{OSL}$ emissions respectively. The pre-dose treatment consisted of a test dose of $0.4 \mathrm{~Gy}$, pre-dose of $10 \mathrm{~Gy}$ and thermal 
activation temperature of $380^{\circ} \mathrm{C}$. The activation temperature of $380^{\circ} \mathrm{C}$, employed in the protocol, step (5), was found to be optimum in the pre-dose activation mechanism in case of both the samples (Koul and Chougaonkar, 2007).

The variation in the sensitization of the fast component of CW-OSL and TL emissions of the Thar and Sludge samples with the re-firing temperatures is shown in Fig. 1a and 1b respectively. The arrows marked in the figure indicate the temperatures at which the samples were pre-fired. The curves consist of three different regions; initial stable portion, middle rising region and finally a falling region. By and large the sensitization curves of TL and OSL have a similar pattern in case of both the samples. The plots do not depict any trend, like monotonous enhancement once re-firing overtakes the firing temperature, which can infer any information about the firing temperature of the specimen.

\section{Response of TL and OSL to heat treatment}

The effective utilization of the (TL/OSL) ratio as a parameter for the firing temperature identification would

a)

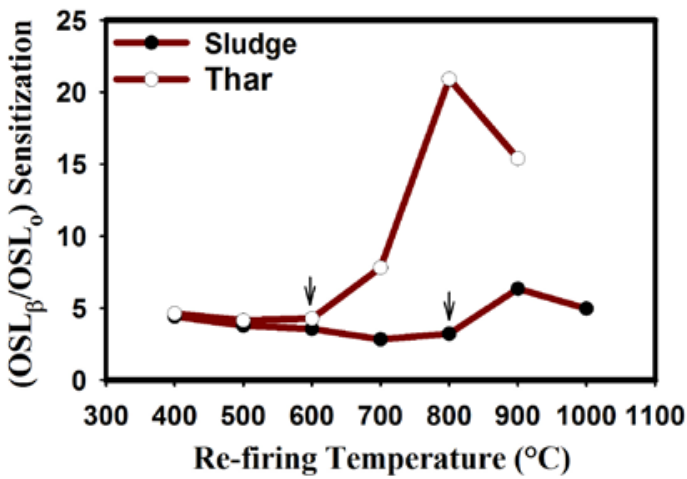

b)

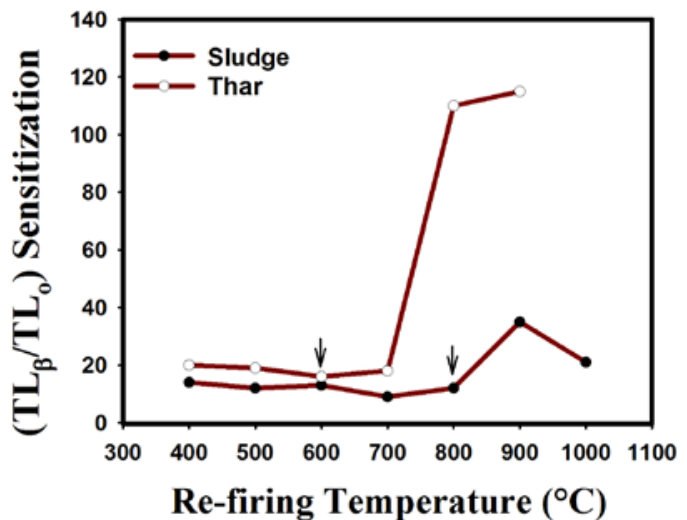

Fig. 1. Measurement of the OSL (a) and TL (b) sensitization of the Thar and Sludge samples after re-firing at various high temperatures $400-900^{\circ} \mathrm{C}$ and $400-1000^{\circ} \mathrm{C}$ in steps of $100^{\circ} \mathrm{C}$ in case of Thar and Sludge samples respectively. The pre-dose treatment consisted of a test dose of $0.4 \mathrm{~Gy}$, pre-dose of $10 \mathrm{~Gy}$ and thermal activation temperature of $380^{\circ} \mathrm{C}$. The arrows point out the firing temperatures experienced by the specimen prior to re-firing, 600 and $800^{\circ} \mathrm{C}$ in case of Thar and Sludge respectively. require to ascertain the behaviour of two signals with high temperature $\left(>500^{\circ} \mathrm{C}\right)$ heating. Though the higher temperature heat treatment has been reported to work in favour of the TL process, it was found necessary to observe the trend in case of the samples studied here (Murray and Wintle, 2000; Chen et al., 2000). This would be necessary for employing this feature in the firing temperature estimation. The study, therefore, comprised of two parts (i) behaviour of TL and OSL emissions with high temperature treatment, i.e. firing and (ii) observation of the (TL/OSL) ratio of the pre-fired specimen with different re-fired temperatures.

\section{Behaviour of (TL/OSL) ratio with firing temperatures}

The samples were fired to different temperatures for one hour in air, in the ranges of 600 and $900^{\circ} \mathrm{C}$ in the case of Thar and 700 and $800^{\circ} \mathrm{C}$ in the case of Sludge. The heating temperature range of $600-900^{\circ} \mathrm{C}$ was chosen with purpose, as this is the typical region of firing encountered by the specimen of interest, ceramics. The luminescence measurements were carried out in the same way as discussed above.

The sensitivity ratio of the two luminescence signals, TL/OSL, at different firing temperatures is depicted in Table 1. The un-sensitized and sensitized modes of luminescence represent the signals recorded with test dose and pre-dose respectively, as mentioned above. As is clear from Table 1, the increase in the firing temperature is found to enhance the TL process in comparison to the OSL sensitization for both the samples. This is true of both, pre-dose sensitized and un-sensitized, luminescence emissions. Also, the larger the difference in the firing temperatures, as is the case with Thar samples here, the higher the variation in the sensitivity ratios. Though, it should be born in mind that these sensitivities also depend on the nature of the specimen. The observations indicated that the magnitude of heating temperature, firing temperature, has a bearing on the ratio of the TL and OSL signals of quartz. So, it seems this parameter might be feasible for the firing temperature identification.

Table 1. Variation of TL and OSL signals with different firing temperatures. This is reflected by the ratio of the two emissions at these heating temperatures. The ratios are shown for both un-sensitized and sensitized signals for both the samples, Thar and Sludge.

\begin{tabular}{lccc}
\hline Sample & $\begin{array}{c}\text { Firing Tempera- } \\
\text { ture } \\
\left({ }^{\circ} \mathrm{C}\right)\end{array}$ & $\begin{array}{c}\text { Un-sensitized } \\
\text { sensitivity ratio } \\
\left(\mathrm{TL}_{\circ} / \mathrm{OSL}_{\mathrm{o}}\right)\end{array}$ & $\begin{array}{c}\text { Sensitized sensi- } \\
\text { tivity ratio } \\
\left(\mathrm{TL}_{\beta} / \mathrm{OSL}_{\beta}\right)\end{array}$ \\
\hline Thar & 600 & 0.72 & 1.67 \\
& 900 & 15.74 & 6.98 \\
\hline Sludge & 700 & 0.46 & 0.75 \\
& 800 & 3.6 & 4.48 \\
\hline
\end{tabular}




\section{Variation of the pre-fired (TL/OSL) ratio with re-firing temperatures}

The sensitivity ratios of TL and OSL signals was derived from the obtained data, measurement of these individual signals with the re-firing temperatures. The ratio curves are depicted in Fig. 2a sensitized and $\mathbf{2 b}$ unsensitized. The overall features of the ratio curves behave in a totally different way for the sensitized and unsensitized emissions. While as the sensitized ratio curves have regions with different trends the un-sensitized curves, more or less, depict a monotonous decrease except for a small shoulder in the case of Thar sample. The TL and OSL ratio curves of the two samples look similar in case of both sensitized and un-sensitized signals.

The sensitized ratio curves, Fig. 2a, do not seem to have any correlation, whatsoever, with the firing temperature experienced by the samples. The increase in both the curves initiates at $700^{\circ} \mathrm{C}$ which does not match with either of the two firing temperatures, 600 and $800^{\circ} \mathrm{C}$, applied in the study. The un-sensitized ratio curves also don't in any way reflect the firing temperature mark; while as it decreases monotonously in case Sludge sam-

a)

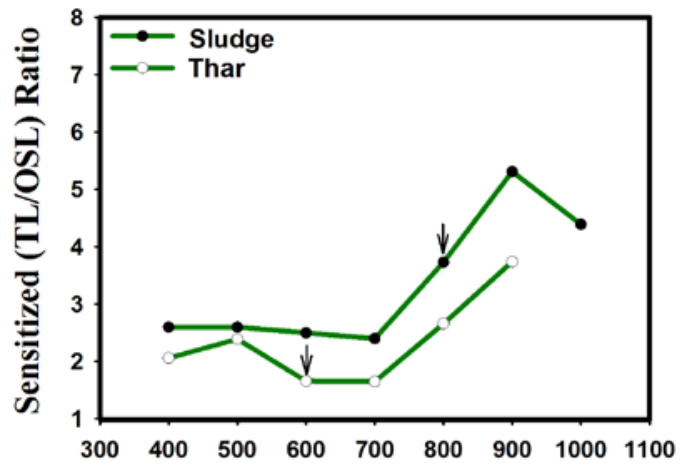

Re-firing Temperature $\left({ }^{\circ} \mathrm{C}\right)$

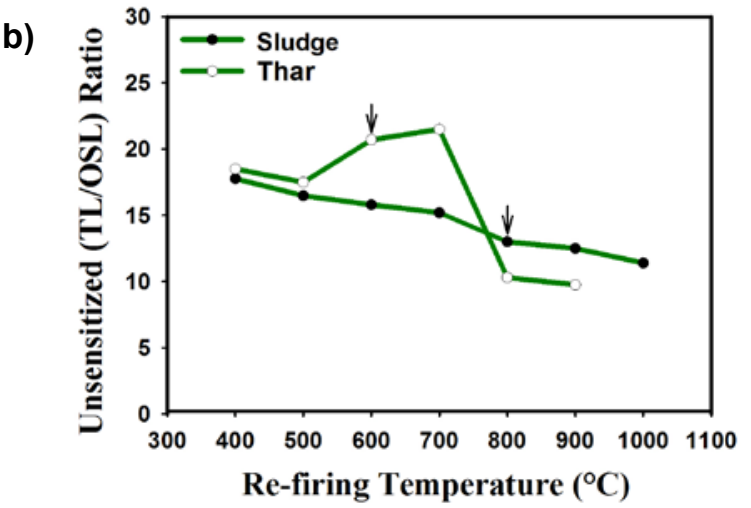

Fig. 2. Variation of the pre-dose signals (a) sensitized and (b) unsensitized ratios of $T L$ and OSL signals with different re-firing temperatures $400-900^{\circ} \mathrm{C}$ and $400-1000^{\circ} \mathrm{C}$ in steps of $100^{\circ} \mathrm{C}$ in case of Thar and Sludge samples respectively. The arrows point out the firing temperature marks experienced by the samples, 600 and $800^{\circ} \mathrm{C}$ in case of Thar and Sludge samples, respectively. The ratios were derived from the data of the Fig. 1. ple the Thar sample demonstrates an ascent at $500^{\circ} \mathrm{C}$ followed by a descent at $700^{\circ} \mathrm{C}$. On a closer look one can observe the similarity in the initial and the tail of the two curves in this plot.

\section{DISCUSSION}

In their studies Sunta and David (1982) observed enhancement in the TL pre-dose sensitization once the refired temperature crossed the firing temperature mark. Thereafter, it increased monotonously in an appreciable way. This way they demonstrated the successful utilization of the technique for the firing temperature evaluation. Watson and Aitken (1985), on the contrary, did not observe any such pattern. Moreover their results lacked mutual consistency, with different samples yielding quite different results.

In order to resolve the incoherence in the two findings mentioned above Koul et al. (1996) examined the validity of the technique using a wide spectral band. This band was chosen to minimize the spectral changes, if any, during the firing process. The results of their studies indicated that the technique might not be generally applicable for firing temperature estimation, same as suggested by Watson and Aitken (1985). One general feature observed in the most of their samples was the desensitization effect with re-firing initiating in the temperature range of $800-900^{\circ} \mathrm{C}$. In fact, this behaviour can also be seen in many of the samples studied by Watson and Aitken (1985). The impact of the heat and irradiation treatments, as incorporated in the pre-dose measurement, on the various impurities present in the quartz lattice was analysed by Koul (2006). Based on this study, it was recognized that it could be the role of the alkali ions which might limit the capacity of the pre-dosed $110^{\circ} \mathrm{C}$ TL peak to remember the firing temperature.

A first look at the sensitization curves of Sludge sample and OSL sensitization curve of Thar may make one to believe that the curves are able to reflect the firing temperature mark. This might happen because the sensitization looks to initiate around the respective firing temperatures. But, the enhancement in the sensitization does not sustain beyond around the $800-900^{\circ} \mathrm{C}$ region, the temperature range where the phase transition occurs in quartz.

Studies carried out to monitor the variation in sensitivity of both the $110^{\circ} \mathrm{C}$ TL and fast component of the CW-OSL emissions with different high heating temperatures have, in general, yielded similar results (BøtterJensen et al., 1995; Schilles et al., 2001; Chen et al., 2001). The sensitivity has been observed to vary appreciably around heating temperatures corresponding to the phase transition of quartz, $\sim 800-900^{\circ} \mathrm{C}$, the region of $\beta$ quartz $-\beta_{2}$ tridymite phase transition of quartz. Based on various techniques TL, LM-OSL, EPR and CW-OSL it has been reported that UV emission at $360 \mathrm{~nm}$ was enhanced by heating to $700^{\circ} \mathrm{C}$ (Poolton et al., 2000; Schilles et al., 2001). So, considering all these observations, 
the overall pattern of the sensitization curves obtained in this study, look to have features similar to those generated by just annealing, firing, of the quartz at higher temperature. The re-firing does not seem to contribute anything significant towards the behaviour of the sensitization curves. The de-sensitization observed here in the temperature range of $800-900^{\circ} \mathrm{C}$ might be attributed to the phase transition of quartz. The sensitization curves seem to carry the signatures of the phase transition.

Our results did support the earlier findings that the higher temperature firing has a bias towards TL mechanism (Table 1). Establishment of firing temperature exploiting this feature was attempted in this study. The sensitized ratio curves, Fig. 2a, more or less behaved in a similar way as the sensitization plots, Fig. 2a. The unsensitized plots, Fig. $\mathbf{2 b}$, pattern was observed to be a mixed one- ratio curve corresponding to Sludge sample decreased monotonously while as the one corresponding to Thar enhanced at $500^{\circ} \mathrm{C}$ and started decreasing at $700^{\circ} \mathrm{C}$. The behaviour of the latter may be explained on the basis of different response of the mechanisms responsible for the OSL and TL emissions to (i) the phase transition occurring in quartz at $573^{\circ} \mathrm{C}$ and the annihilation of competitive centre, $\mathrm{E}_{1}$ ', active in this heating temperature region (Koul, Submitted). The impact of the firing temperature was presumed to get reflected in the sensitization ratio curves once the re-firing temperature gets over the firing temperature value. The hypothesis conceived was (i) the firing at certain high temperature will establish a certain value of the (TL/OSL) ratio, (ii) re-firing at temperatures lower than the firing temperature will not alter the ratio as the specimen has already experienced temperature higher than these values and (iii) once the re-firing exceeds the firing temperature the ratio would be reset and result in enhancing the (TL/OSL) sensitization further. However, the results did not seem to follow this hypothesis.

\section{CONCLUSION}

The results suggested that the methods based on the pre-dose sensitization of the OSL emission, like its $110^{\circ} \mathrm{C}$ TL counterpart, might not be able to identify the firing temperature mark. The firing of the sample at higher temperatures was observed to favour the TL process. The firing temperature estimation, utilizing the ratio of the TL and OSL signals, again, could not reveal the firing temperature. The measurements carried out seem to be influenced by the phase transition phenomenon of quartz.

\section{ACKNOWLEDGEMENTS}

We thank Mr. R. Koul, Head, ApSD, BARC and Mr. V.D. Puranik, Head, EAD, BARC for their support during this study. We also thank Dr. P.G. Benny, Radiation Technology development Section, HIRUP, BARC, Baroda for providing us the samples and Dr. John D. John,
MSD, BARC for carrying out the heat treatments of the samples.

\section{REFERENCES}

Aitken MJ, 1985. Thermoluminescence Dating. Academic Press, London: $359 \mathrm{pp}$

Aitken MJ and Smith BW, 1988. Optical dating: recuperation after bleaching. Quaternary Science Revews 7(3-4): 387-393, DOI 10.1016/0277-3791(88)90034-0.

Bøtter-Jensen L, Agersnap LN, Mejdahl V, Poolton NRJ, Morris MF and Mckeever SWS, 1995. Luminescence sensitivity changes in quartz as a result of annealing. Radiation Measurements 24(4): 535-541, DOI 10.1016/1350-4487(95)00006-Z.

Chen G, Li SH and Murray AS, 2000. Study of the $110^{\circ} \mathrm{C}$ TL peak sensitivity in optical dating of quartz. Radiation Measurements 32(5-6): 641-645, DOI 10.1016/S1350-4487(00)00129-3.

Chen G, Murray AS and Li SH, 2001. Effect of the heating on the quartz dose response curve. Radiation Measurements 33(1): 59-63, DOI 10.1016/S1350-4487(00)00134-7.

Franklin AD, Prescott JR and Scholefield RB, 1995. The mechanism of thermoluminescence in an Australian sedimentary quartz. Journal of Luminescence 63(5-6): 317-326, DOI 10.1016/00222313(94)00068-N

Godfrey-Smith DI and Ilani S, 2004. Past thermal history of goethite and hematite fragments from Qafzeh Cave deduced from thermal activation characteristics of the $110^{\circ} \mathrm{C}$ TL peak of enclosed quartz grains. Revue d'archeometrie 28: 185-190.

Goksu HY, Weiser A and Regulla DF, $1989.110^{\circ} \mathrm{C}$ TL peak records the ancientheat treatment of flint. Ancient TL 7 15-17.

Jones CHW, Dombsky M and Skinner AF, 1979. Effect of heat treatment on the iron-57 Mossbauer spectrum. Journal de Physique. Colloques 2: 46-51.

Koul DK, 2006. Role of alkali-ions in limiting the capacity of the $110^{\circ} \mathrm{C}$ peak of quartz to remember the firing temperature. Applied Radiation and Isotopes 64(1): 110-115, DOI 10.1016/j.apradiso.2005.07.008.

Koul DK, Submitted. Co-relation of the pre-dosed TL and OSL emissions of quartz. Radiation Measurements.

Koul DK and Chougaonkar MP, 2007. Pre-dose phenomenon in the OSL signal of quartz. Radiation Measurements 42(8): 1265-1272, DOI 10.1016/j.radmeas.2007.04.001.

Koul DK, Singhvi AK, Nambi KSV, Bhat CL, Gupta PK, 1996. Feasibility of estimating firing temperature using the $110^{\circ} \mathrm{C}$ peak of quartz. Applied Radiation and Isotopes 47(2): 191-195, DOI 10.1016/0969-8043(96)82846-6.

Lahaye C, Godfrey-Smith DI, Guibert P and Bechtel F, 2006. Equivalent thermal history $\left(\mathrm{H}_{\mathrm{E}}\right)$ of ferruginous sandstones based on the thermal activation characteristics of quartz. Radiation Measurements 41(7-8): 995-1000, DOI 10.1016/j.radmeas.2006.04.021.

Li SH, 2002. Luminescence sensitivity changes of quartz by bleaching, annealingand UV exposure. Radiation Effects and Defects in Solids $157: 357-364$.

Murray AS and Roberts RG, 1998. Measurement of the equivalent dose in quartz using a regenerative-dose single-aliquot protocol. Radiation Measurements 29(5): 503-515, DOI 10.1016/S13504487(98)00044-4.

Murray AS and Wintle AG, 2000. Luminescence dating of quartz using an improved single-aliquot regenerative-dose protocol. Radiation Measurements 32(1): 57-73, DOI 10.1016/S1350-4487(99)00253$\mathrm{X}$.

Polymeris GS, Sakalis A, Papadopoulou D, Dallas G, Kitis G and Tsirliganis NC, 2007. Firing temperature of pottery using TL and OSL techniques. Nuclear Instruments and Methods in Physics Research Section A 580(1): 747-750, DOI 10.1016/j.nima.2007.05.139.

Poolton NRJ, Smith GM, Riedi PC, Bulur E, Bøtter-Jensen L, Murray AS and Adrian M, 2000. Luminescence sensitivity changes in natural quartz induced by high temperature annealing: a high frequen- 
cy EPR and OSL study. Journal of Physics D: Applied Physics 33(8): 1007-1017, DOI 10.1088/0022-3727/33/8/318.

Robins GV, Seeley NJ, McNeil DAC and Symons MCR, 1978. Identification of ancient heat treatment in flint artefacts by ESR spectroscopy. Nature 276: 703-704, DOI 10.1038/276703a0.

Roque C, Guibert P, Vartanian E, Vieillevigne E and Bechtel F, 2004. Changes in luminescence properties induced by thermal treatments; a case study at Sipan and Trujillo Moche sites (Peru). Radiation Measurements 38(1): 119-126, DOI 10.1016/S13504487(03)00249-X.

Schilles T, Poolton NRJ, Bulur E, Bøtter-Jensen L, Murray AS, Smith GM, Riedi PC and Wagner GA, 2001. A multi-spectroscopic study of luminescence sensitivity changes in natural quartz induced by high temperature annealing. Journal of Physics D: Applied Physics 34(5): 722-731, DOI 10.1088/0022-3727/34/5/310.
Sunta CM and David M, 1982. Firing temperature of pottery from predose sensitization of TL. PACT 6: 460-467.

Valladas H, 1983. Estimation de la temperature de chauffe de silexprehistoriques par leur thermoluminescence. Comptes Rendus de l'Académie des Sciences 296: 993-996 (in French).

Watson IA and Aikten MJ, 1985. Firing temperature analysis using the $110^{\circ} \mathrm{C}$ TL peak of quartz. Nuclear Track 10(4-6): 517-520, DOI 10.1016/0735-245X(85)90052-3.

Weymouth TW and Mandeville M, 1975. An X-ray diffraction study of heat treated cherts and its archaeological implications. Archaeometry 17(1): 61-66. DOI 10.1111/j.1475-4754.1975.tb00115.x.

Zimmerman J, 1971. The radiation - induced increase of the $100^{\circ} \mathrm{C}$ thermoluminescence sensitivity of fired quartz. Journal of Physics C Solid State Physics 4(18): 3265-3276, DOI 10.1088/00223719/4/18/032. 